TRANSACTIONS OF THE

AMERICAN MATHEMATICAL SOCIETY

Volume 358, Number 1, Pages 115-129

S 0002-9947(05)04079-1

Article electronically published on August 25, 2005

\title{
MORSE THEORY FROM AN ALGEBRAIC VIEWPOINT
}

\author{
EMIL SKÖLDBERG
}

\begin{abstract}
Forman's discrete Morse theory is studied from an algebraic viewpoint, and we show how this theory can be extended to chain complexes of modules over arbitrary rings. As applications we compute the homologies of a certain family of nilpotent Lie algebras, and show how the algebraic Morse theory can be used to derive the classical Anick resolution as well as a new two-sided Anick resolution.
\end{abstract}

\section{INTRODUCTION}

Forman's discrete Morse theory [For98 has been successfully applied to problems in combinatorial topology, e.g. to study the homotopy type and homology of graph complexes (for examples, see Babson et al. BBL:99, Jonsson Jon03, and Shareshian [Sha01]).

The idea in discrete Morse theory is to reduce the number of cells in a CWcomplex without changing the homotopy type. This new complex is constructed via a discrete Morse function, or equivalently (see Chari Cha00]), via a certain partial matching of the cells. In this note we derive an algebraic version of this theory, where we consider chain complexes of modules with a fixed decomposition into direct summands; these summands play the role of the cells in the topological situation. Given a partial matching of the summands fulfilling certain properties, we can then construct a smaller complex which is homotopy equivalent to the original complex.

The theory is then applied to compute the Betti numbers of the nilpotent Lie algebra that has basis elements $z, x_{1}, \ldots, x_{n}, y_{1}, \ldots, y_{n}$ and the only non-vanishing Lie brackets being $\left[z, x_{i}\right]=y_{i}$, over a field of characteristic 2 . The Betti numbers for this Lie algebra over a field of characteristic 0 have been earlier obtained by Armstrong, Cairns, and Jessup ACJ97. As another application, we see how we can construct the Anick resolution of the ground field $k$ over an augmented $k$ algebra $A$ by applying the theory. Finally we obtain a two-sided version of the Anick resolution, that is a free $A \otimes_{k} A^{\mathrm{op}}$-resolution of $A$, which is a new result.

Batzies and Welker have used discrete Morse theory in an algebraic setting in BW02 to construct minimal free resolutions of generic and shellable monomial ideals, but by using that the exactness of the complex they construct is equivalent to the contractibility of a certain CW-complex, which enables them to use the techniques from discrete Morse theory.

Another, less general, approach to constructing an algebraic version of Forman's theory is due to Jakob Jonsson [Jon03.

Received by the editors August 4, 2003.

2000 Mathematics Subject Classification. Primary 16E05; Secondary 16E40, 17 B56.

(C) 2005 American Mathematical Society Reverts to public domain 28 years from publication 
After the completion of this work, the author learned that Jöllenbeck and Welker have obtained similar results in JW05.

\section{Matchings AND SPlitTing Homotopies}

Let $R$ be a ring with unit. By a based complex of $R$-modules we mean a chain complex $\mathbf{K}$ of (left or right) $R$-modules together with a direct sum decomposition $K_{n}=\bigoplus_{\alpha \in I_{n}} K_{\alpha}$, where $\left\{I_{n}\right\}$ is a family of mutually disjoint index sets. When $\alpha \in \bigcup_{n} I_{n}$ we write $\alpha^{(n)}$ to indicate that $\alpha \in I_{n}$.

For $f: \bigoplus_{n} K_{n} \rightarrow \bigoplus_{n} K_{n}$ a graded map, we write $f_{\beta, \alpha}$ for the component of $f$ going from $K_{\alpha}$ to $K_{\beta}$, i.e.,

$$
f_{\beta, \alpha}: K_{\alpha} \longrightarrow K_{m} \stackrel{f}{\longrightarrow} K_{n} \longrightarrow K_{\beta},
$$

with the obvious inclusion $K_{\alpha} \longrightarrow K_{m}$ and projection $K_{n} \longrightarrow K_{\beta}$. Note the order of the indices, chosen to agree with the composition of functions.

Given a based complex $\mathbf{K}$ we construct a digraph $G_{\mathbf{K}}$ with vertex set $V=\bigcup_{n} I_{n}$ and with a directed edge $\alpha \rightarrow \beta$ whenever the component $d_{\beta, \alpha}$ is non-zero.

A partial matching on a digraph $D=(V, E)$ is a subset $A$ of the edges $E$ such that no vertex is incident to more than one edge in $A$. In this situation we define the new digraph $D^{A}=\left(V, E^{A}\right)$ to be the digraph obtained from $D$ by reversing the direction of each arrow in $A$, i.e.

$$
E^{A}=(E \backslash A) \cup\{\beta \rightarrow \alpha \mid \alpha \rightarrow \beta \in A\} .
$$

Recall that a partial order on a set $P$ is well founded if there is no strictly descending infinite sequence in $P$.

We call a partial matching $M$ on the digraph $G_{\mathbf{K}}$ a Morse matching if, for each edge $\alpha \rightarrow \beta$ in $M$, the corresponding component $d_{\beta, \alpha}$ is an isomorphism, and furthermore there is a well-founded partial order $\prec$ on each $I_{n}$ such that $\gamma \prec \alpha$ whenever there is a path $\alpha^{(n)} \rightarrow \beta \rightarrow \gamma^{(n)}$ in $G_{\mathrm{K}}^{M}$.

A vertex in $G_{\mathbf{K}}^{M}$ that is unmatched, i.e. not incident to an edge in $M$, is called $M$-critical. We will use the notation $M^{0}$ for the set of $M$-critical vertices, and we define sets $M^{-}$and $M^{+}$by

$$
\begin{aligned}
& M^{-}=\{\alpha \mid \beta \rightarrow \alpha \in M \text { for some } \beta\}, \\
& M^{+}=\{\alpha \mid \alpha \rightarrow \gamma \in M \text { for some } \gamma\},
\end{aligned}
$$

and we will also use the notation $M_{n}^{0}, M_{n}^{-}$and $M_{n}^{+}$for the sets $M^{0} \cap I_{n}, M^{-} \cap I_{n}$ and $M^{+} \cap I_{n}$.

Whenever the digraph $G_{\mathbf{K}}$ is finite, the following lemma gives a simpler characterisation of the Morse matchings.

Lemma 1. Let $\mathbf{K}$ be a based complex such that $G_{\mathbf{K}}$ is a finite directed graph, and let $M$ be a partial matching on $G_{\mathbf{K}}$ such that $d_{\beta, \alpha}$ is an isomorphism whenever $\alpha \rightarrow \beta$ is in $M$. Then $M$ is a Morse matching if and only if $G_{\mathbf{K}}^{M}$ has no directed cycles.

Proof. Suppose that $G_{\mathbf{K}}^{M}$ has no directed cycles. For a vertex $u$ of $G_{\mathbf{K}}^{M}$ we define

$$
\ell(u)=\max \left\{s \mid u^{(n)}=u_{0}^{(n)} u_{1} u_{2}^{(n)} \cdots u_{s-1} u_{s}^{(n)} \text { directed path in } G_{\mathbf{K}}^{M}\right\} ;
$$


$\ell(u)$ is finite since there are no directed cycles in the digraph. Now let $u^{(n)} \prec v^{(n)}$ if $\ell(u)<\ell(v)$; this is a well founded partial order, which implies that $M$ is a Morse matching.

For the converse, we assume that there is a directed cycle $u_{1} u_{2} \cdots u_{n}$ with $u_{n}=$ $u_{1}$ in $G_{\mathbf{K}}^{M}$. It follows from the definition of a matching that, for all $i$, at most one of $u_{i} u_{i+1}$ and $u_{i+1} u_{i+2}$ is the reverse of an edge in $M$. But since $u_{n}=u_{1}$ it must be the case that the reverse of every second edge in the cycle is in $M$. Thus, if $M$ would be a Morse matching we would have $u_{1}=u_{n} \prec u_{1}$, which is an absurdity.

Example 1. Let $\Delta$ be a finite abstract simplicial complex on the vertex set $[n]$. The chain complex $C(\Delta)$ associated to $\Delta$ now has a natural basis consisting of the simplices of $\Delta$ which gives the structure of a based complex. We can now construct a matching $M$ on $C(\Delta)$ by

$$
M=\{\sigma \rightarrow \sigma \backslash\{1\} \mid \sigma \in \Delta, 1 \in \sigma\} .
$$

It is easy to see that there are no cycles in the graph $G_{C(\Delta)}^{M}$, which implies that $M$ is a Morse matching. The $M$-critical vertices are the simplices $\sigma$ such that $1 \notin \sigma$ and $\sigma \cup\{1\} \notin \Delta$.

Given a Morse matching $M$ on the based complex $\mathbf{K}$ we will define a graded map $\phi: \bigoplus_{n} K_{n} \rightarrow \bigoplus_{n} K_{n}$ of degree 1 , and then show that it is a splitting homotopy, a concept introduced by Barnes and Lambe BL91. Recall that a splitting homotopy is a map $\phi$ as above that satisfies

$$
\begin{gathered}
\phi^{2}=0, \\
\phi d \phi=\phi .
\end{gathered}
$$

Let us now define the mapping $\phi$ inductively as follows: If $\alpha$ is minimal with respect to $\prec$ and $x \in K_{\alpha}$, let

$$
\phi(x)= \begin{cases}d_{\alpha, \beta}^{-1}(x) & \text { if } \beta \rightarrow \alpha \in M \text { for some } \beta, \\ 0 & \text { otherwise. }\end{cases}
$$

If $\alpha$ is not minimal with respect to $\prec$ and $x \in K_{\alpha}$, let

$$
\phi(x)= \begin{cases}d_{\alpha, \beta}^{-1}(x)-\sum_{\substack{\beta \rightarrow \gamma \\ \gamma \neq \alpha}} \phi d_{\gamma, \beta} d_{\alpha, \beta}^{-1}(x) & \text { if } \beta \rightarrow \alpha \in M \text { for some } \beta, \\ 0 & \text { otherwise. }\end{cases}
$$

This is well defined since for all $\gamma$ that appear in the last sum, we have $\gamma \prec \alpha$.

Lemma 2. Let $M$ be a Morse matching on the based complex $\mathbf{K}$. The map $\phi$ is then a splitting homotopy.

Proof. That $\phi^{2}=0$ follows directly from the matching properties, and we will prove that $\phi d \phi=\phi$ by induction over $\prec$. Consider the case where $\alpha$ is minimal with respect to $\prec$. If $\alpha \notin M^{-}$, then for $x \in K_{\alpha}$

$$
\phi d \phi(x)=0=\phi(x) .
$$

If $\alpha \in M^{-}$with $\beta \rightarrow \alpha \in M$, then

$$
\phi d \phi(x)=\phi d d_{\alpha, \beta}^{-1}(x)=\phi d_{\alpha, \beta} d_{\alpha, \beta}^{-1}(x)=\phi(x) .
$$


Now consider the case where $\alpha$ is not minimal. We still have that $\phi d \phi(x)=0=\phi(x)$ when $\alpha \notin M^{-}$, so assume that $\alpha \in M^{-}$with $\beta \rightarrow \alpha \in M$. We then get

$$
\begin{aligned}
\phi d \phi(x) & =\phi d\left(d_{\alpha, \beta}^{-1}(x)-\sum_{\substack{\beta \rightarrow \gamma \\
\gamma \neq \alpha}} \phi d_{\gamma, \beta} d_{\alpha, \beta}^{-1}(x)\right) \\
& =\phi d d_{\alpha, \beta}^{-1}(x)-\sum_{\substack{\beta \rightarrow \gamma \\
\gamma \neq \alpha}} \phi d \phi d_{\gamma, \beta} d_{\alpha, \beta}^{-1}(x) \\
& =\phi d_{\alpha, \beta} d_{\alpha, \beta}^{-1}(x)+\sum_{\substack{\beta \rightarrow \gamma \\
\gamma \neq \alpha}} \phi d_{\gamma, \beta} d_{\alpha, \beta}^{-1}(x)-\sum_{\substack{\beta \rightarrow \gamma \\
\gamma \neq \alpha}} \phi d_{\gamma, \beta} d_{\alpha, \beta}^{-1}(x) \\
& =\phi(x) .
\end{aligned}
$$

The second-to-last equality follows from the fact that, for all $\gamma$ that appear in the sum, we have $\gamma \prec \alpha$, so by induction $\phi d \phi(y)=y$ for all $y \in K_{\gamma}$.

Let us now define the map $\pi: \mathbf{K} \rightarrow \mathbf{K}$ by

$$
\pi=\mathrm{id}-(\phi d+d \phi)
$$

We can then formulate the algebraic version of of Forman's theorem, For98, Theorem 8.2]. Namely that only the modules corresponding to the $M$-critical vertices contribute to the homology of the complex; see also [Jon03, Theorem 6.1]. The proof uses two technical lemmas which are stated and proved after the theorem.

Theorem 1. Let $M$ be a Morse matching on the based complex $\mathbf{K}$. Then the complexes $\mathbf{K}$ and $\pi(\mathbf{K})$ are homotopy equivalent. Furthermore we have for each $n$ an isomorphism of modules:

$$
\pi\left(K_{n}\right) \simeq \bigoplus_{\alpha \in M_{n}^{0}} K_{\alpha}
$$

Proof. It is easy to see that $\pi$ is a chain map, and from the definition it is clear that $\pi$ is homotopic to the identity map, so the complexes $\mathbf{K}$ and $\pi(\mathbf{K})$ are homotopy equivalent.

The next thing we have to do is to prove that

$$
\pi(\mathbf{K})=\pi\left(\bigoplus_{\gamma \in M^{0}} K_{\gamma}\right)
$$

by showing that $\pi\left(K_{\alpha}\right) \subseteq \pi\left(\bigoplus_{\gamma \in M^{0}} K_{\gamma}\right)$ for all $\alpha$ by induction on $\prec$.

When $\alpha$ is minimal, either $\alpha \in M^{0}$ in which case there is nothing to prove, or $\alpha \notin M^{0}$ in which case $\pi(x)=0$ for $x \in K_{\alpha}$ by Lemmas 3 and 4 .

When $\alpha$ is non-minimal, there is still nothing to prove when $\alpha \in M^{0}$, so assume that $\alpha \notin M^{0}$ with $x \in K_{\alpha}$. Then, by Lemmas 3 and 4, there is a set $J$ with $\gamma \prec \alpha$ for all $\gamma \in J$, and

$$
\pi(x)=\pi^{2}(x)=\pi\left(\sum_{\gamma \in J} y_{\gamma}\right)=\sum_{\substack{\gamma \in J \\ \gamma \in M^{0}}} \pi\left(y_{\gamma}\right)+\sum_{\substack{\gamma \in J \\ \gamma \notin M^{0}}} \pi\left(y_{\gamma}\right),
$$

where $y_{\gamma} \in K_{\gamma}$. By induction this proves (1).

The next step is to show that

$$
\pi: \bigoplus_{\alpha \in M_{n}^{0}} K_{\alpha} \longrightarrow K_{n}
$$


is injective. By Lemmas 3 and 4 , we can conclude that for $x \in K_{\alpha}$ with $\alpha \in M^{0}$,

$$
\pi(x)=x+\sum_{\gamma \in J} y_{\gamma}
$$

where $y_{\gamma} \in K_{\gamma}$ and $\gamma \prec \alpha$ for all $\gamma \in J$, and this proves the injectivity.

Lemma 3. Let $\mathbf{K}$ be a based complex with a Morse matching $M$ on $G_{\mathbf{K}}$ and corresponding splitting homotopy $\phi$. When $x_{\alpha} \in K_{\alpha}$,

$$
d \phi\left(x_{\alpha}\right)= \begin{cases}x_{\alpha}+\sum_{\beta \prec \alpha} y_{\beta} & \text { if } \alpha \in M^{-}, \\ 0 & \text { otherwise, }\end{cases}
$$

where $y_{\beta} \in K_{\beta}$ for all $\beta$.

Proof. We will prove the statement by induction over $\prec$. Consider the case when $\alpha$ is minimal. If $\alpha \notin M^{-}$it is clear that $d \phi\left(x_{\alpha}\right)=0$, so assume that $\alpha \in M^{-}$with $\beta \rightarrow \alpha \in M$. In this case we have

$$
d \phi\left(x_{\alpha}\right)=d d_{\alpha, \beta}^{-1}\left(x_{\alpha}\right)=d_{\alpha, \beta} d_{\alpha, \beta}^{-1}\left(x_{\alpha}\right)=x_{\alpha} .
$$

In the case when $\alpha$ is not minimal, it is still the case that $d \phi(x)=0$ for $\alpha \notin M^{-}$, so if we assume that $\alpha \in M^{-}$with $\beta \rightarrow \alpha \in M$, we get

$$
\begin{aligned}
d \phi\left(x_{\alpha}\right) & =d\left(d_{\alpha, \beta}^{-1}\left(x_{\alpha}\right)-\sum_{\substack{\beta \rightarrow \gamma \\
\gamma \neq \alpha}} \phi d_{\gamma, \beta} d_{\alpha, \beta}^{-1}\left(x_{\alpha}\right)\right) \\
& =x_{\alpha}+\sum_{\substack{\beta \rightarrow \gamma \\
\gamma \neq \alpha}} d_{\gamma, \beta} d_{\alpha, \beta}^{-1}\left(x_{\alpha}\right)-d \phi \sum_{\substack{\beta \rightarrow \gamma \\
\gamma \neq \alpha}} d_{\gamma, \beta} d_{\alpha, \beta}^{-1}\left(x_{\alpha}\right) .
\end{aligned}
$$

For each term that appears in the last two sums we have that $\gamma \prec \alpha$.

Lemma 4. Let $\mathbf{K}$ be a based complex with a Morse matching $M$ on $G_{\mathbf{K}}$ and corresponding splitting homotopy $\phi$. When $x_{\alpha} \in K_{\alpha}$,

$$
\phi d\left(x_{\alpha}\right)= \begin{cases}x_{\alpha} & \text { if } \alpha \in M^{+}, \\ \sum_{\beta \prec \alpha} y_{\beta} & \text { otherwise, }\end{cases}
$$

where $y_{\beta} \in K_{\beta}$ for all $\beta$.

Proof. Again we prove the statement by induction over $\prec$. Consider the case when $\alpha$ is minimal. When $\alpha \in M^{+}$with, say, $\alpha \rightarrow \beta \in M$, then

$$
\phi d\left(x_{\alpha}\right)=\phi d_{\beta, \alpha}\left(x_{\alpha}\right)+\sum_{\substack{\alpha \rightarrow \gamma \\ \alpha \neq \beta}} \phi d_{\gamma, \alpha}\left(x_{\alpha}\right)=d_{\beta, \alpha}^{-1} d_{\beta, \alpha}\left(x_{\alpha}\right)=x_{\alpha} .
$$

When $\alpha \notin M^{+}$, we get

$$
\phi d\left(x_{\alpha}\right)=\phi \sum_{\alpha \rightarrow \beta} d_{\beta, \alpha}\left(x_{\alpha}\right)=0
$$


since $\alpha$ is minimal. Now consider the case when $\alpha$ is non-minimal; when $\alpha \in M^{+}$ with $\alpha \rightarrow \beta \in M$,

$$
\begin{aligned}
\phi d\left(x_{\alpha}\right) & =\phi d_{\beta, \alpha}\left(x_{\alpha}\right)+\sum_{\substack{\alpha \rightarrow \gamma \\
\alpha \neq \beta}} \phi d_{\gamma, \alpha}\left(x_{\alpha}\right) \\
& =d_{\beta, \alpha}^{-1} d_{\beta, \alpha}\left(x_{\alpha}\right)-\sum_{\substack{\alpha \rightarrow \gamma \\
\alpha \neq \beta}} \phi d_{\gamma, \alpha}\left(x_{\alpha}\right)+\sum_{\substack{\alpha \rightarrow \gamma \\
\alpha \neq \beta}} \phi d_{\gamma, \alpha}\left(x_{\alpha}\right)=x_{\alpha} .
\end{aligned}
$$

When $\alpha \notin M^{+}$,

$$
\phi d\left(x_{\alpha}\right)=\phi \sum_{\alpha \rightarrow \beta} d_{\beta, \alpha}\left(x_{\alpha}\right)=\sum_{\substack{\alpha \rightarrow \beta \\ \gamma \rightarrow \beta \in M}} d_{\beta, \gamma}^{-1} d_{\beta, \alpha}\left(x_{\alpha}\right)-\phi \sum_{\substack{\alpha \rightarrow \beta \\ \gamma \rightarrow \beta \in M}} \sum_{\substack{\gamma \rightarrow \delta \\ \delta \neq \beta}} d_{\delta, \gamma} d_{\beta, \gamma}^{-1} d_{\beta, \alpha}\left(x_{\alpha}\right) .
$$

Clearly, for all $\delta$ in the last sum we have $\delta \prec \alpha$.

The following is a useful corollary whose homotopical counterpart has been used to show that certain classes of graph complexes have the homotopy type of a wedge of spheres; see [BBL:99], Jon03, and [Sha01].

Corollary 1. If $M$ is a Morse matching on $G_{\mathbf{K}}$, such that $M^{0}$ is concentrated in degree $n$, then

$$
H_{i}(\mathbf{K})= \begin{cases}\bigoplus_{\alpha \in M_{n}^{0}} K_{\alpha} & \text { if } i=n, \\ 0 & \text { otherwise. }\end{cases}
$$

Proof. It follows from Theorem 1 that $H_{i}(\mathbf{K})=H_{i}(\pi(\mathbf{K}))$, but the differential on $\pi(\mathbf{K})$ is identically 0 , and $\pi(\mathbf{K}) \simeq \bigoplus_{\alpha \in M^{0}} K_{\alpha}$.

In some situations, the direct sum of the components corresponding to the $M$ critical vertices actually form a subcomplex of $\mathbf{K}$; in this case we get a particularly nice description of $\pi(\mathbf{K})$ :

Corollary 2. If $\mathbf{K}$ is a based complex and $M$ is a Morse matching on $G_{\mathbf{K}}$ such that $\bigoplus_{\alpha \in M^{0}} K_{\alpha}$ form a subcomplex $\mathbf{L}$ of $\mathbf{K}$, then $\mathbf{L}$ and $\mathbf{K}$ are homotopy equivalent.

Proof. Let $x \in K_{\alpha}$ with $\alpha \in M^{0}$. It is then clear that $d \phi(x)=0$, and since $d(x)=\sum_{\beta} y_{\beta}$ with $y_{\beta} \in K_{\beta}$ with all $\beta \in M^{0}$, we get that $\phi d(x)=0$ as well. Thus $\pi(x)=x$, so we can conclude that $\pi(\mathbf{K})=\mathbf{L}$.

From Theorem 1 we can conclude that we can always define a differential on the graded module $\bigoplus_{\alpha \in M^{0}} K_{\alpha}$ such that the resulting complex is isomorphic to $\pi(\mathbf{K})$. The task of defining such a differential is next at hand.

Let us define the complex $(\mathbf{C}, \tilde{d})$ by $C_{n}=\bigoplus_{\alpha \in M_{n}^{0}} K_{\alpha}$. Let $\rho$ be the projection map

$$
\rho: \mathbf{K}=\bigoplus_{\alpha} K_{\alpha} \longrightarrow \bigoplus_{\alpha \in M^{0}} K_{\alpha}
$$

We then define the differential $\tilde{d}$ by

$$
\tilde{d}=\rho(d-d \phi d) .
$$

Theorem 2. The complex $\mathbf{C}$ is homotopy equivalent to the complex $\mathbf{K}$. 
Proof. From Lemmas 3 and 4 we see that the map $\rho \pi$ is the identity on the graded module $\bigoplus_{n} C_{n}$. Furthermore, if we let $y$ be an arbitrary element of $\pi(\mathbf{K})$, we can then write $y=\pi(x)$ with $x \in \bigoplus_{\alpha \in M^{0}} K_{\alpha}$, and $\pi \rho(y)=\pi \rho \pi(x)=\pi(x)=y$, so $\rho$ and $\pi$ are mutual inverses of graded modules between $\pi(\mathbf{K})$ and $\mathbf{C}$. Thus we can define a differential $\tilde{d}$ on $\mathbf{C}$ making it isomorphic to $\pi(\mathbf{K})$, by $\tilde{d}=\rho d \pi$. If we analyse this expression, we see that

$$
\rho d \pi=\rho d(\mathrm{id}-d \phi-\phi d)=\rho(d-d \phi d) .
$$

Since $\pi(\mathbf{K})$ and $\mathbf{K}$ are homotopy equivalent, this concludes the proof.

We can also give a non-recursive description of the differential, similar to Forman's description in terms of gradient paths. For vertices $\alpha$ and $\beta$ in adjacent degrees, we let $\Gamma_{\beta, \alpha}$ be the set of directed 'zig-zag' paths in the graph $G_{\mathbf{K}}^{M}$ of the form

$$
\alpha=\sigma_{1} \sigma_{2} \ldots \sigma_{2 k-1} \sigma_{2 k}=\beta,
$$

where all $\sigma_{2 k+1}$ lie in the same degree as $\alpha$ and all $\sigma_{2 k}$ lie in the same degree as $\beta$. For a directed path $\gamma \in \Gamma_{\beta^{(n)}, \alpha^{(n+1)}}$ we define $m(\gamma)$ by

$$
m(\gamma)=(-1)^{k-1} d_{\sigma_{2 k}^{(n)}, \sigma_{2 k-1}^{(n+1)}} d_{\sigma_{2 k-2}^{(n)}, \sigma_{2 k-1}^{(n+1)}}^{-1} \cdots d_{\sigma_{2}^{(n)}, \sigma_{3}^{(n+1)}}^{-1} d_{\sigma_{2}^{(n)}, \sigma_{1}^{(n+1)}},
$$

and similarly for $\gamma \in \Gamma_{\beta^{(n+1)}, \alpha^{(n)}}$,

$$
m(\gamma)=(-1)^{k-1} d_{\sigma_{2 k-1}^{(n)}, \sigma_{2 k}^{(n+1)}}^{-1} d_{\sigma_{2 k-1}^{(n)}, \sigma_{2 k-2}^{(n+1)}} \cdots d_{\sigma_{3}^{(n)}, \sigma_{2}^{(n+1)}} d_{\sigma_{1}^{(n)}, \sigma_{2}^{(n+1)}}^{-1} .
$$

Using the following lemma, we can describe the differential in $\mathbf{C}$.

Lemma 5. For $x \in K_{\alpha^{(n)}}$ we have

$$
\phi(x)=\sum_{\sigma^{(n+1)}} \sum_{\gamma \in \Gamma_{\sigma, \alpha}} m(\gamma)(x) .
$$

Proof. We proceed by induction. If $\alpha$ is minimal with respect to $\prec$ and $x \in K_{\alpha}$, it is easy to see that because $\phi(x)=d_{\alpha, \beta}^{-1}(x)$ or $\phi(x)=0$ depending on whether $\alpha \in M^{-}$or not, the equality holds in these cases. If $\alpha$ is not minimal, we still have that $\phi(x)=0$ whenever $\alpha \notin M^{-}$, so assume that $\alpha \in M^{-}$. In this case

$$
\begin{aligned}
\phi(x) & =d_{\alpha, \beta}^{-1}(x)-\sum_{\substack{\beta \rightarrow \delta \\
\delta \neq \alpha}} \phi d_{\delta, \beta} d_{\alpha, \beta}^{-1}(x) \\
& =d_{\alpha, \beta}^{-1}(x)-\sum_{\substack{\beta \rightarrow \delta \\
\delta \neq \alpha}} \sum_{\sigma^{(n+1)}} \sum_{\gamma \in \Gamma_{\sigma, \delta}} m(\gamma) d_{\delta, \beta} d_{\alpha, \beta}^{-1}(x) \\
& =\sum_{\sigma^{(n+1)}} \sum_{\gamma \in \Gamma_{\sigma, \alpha}} m(\gamma)(x) .
\end{aligned}
$$

Corollary 3. For $x \in K_{\alpha^{(n)}}$ we have

$$
\tilde{d}(x)=\sum_{\sigma^{(n-1)}} \sum_{\gamma \in \Gamma_{\sigma, \alpha}} m(\gamma)(x) .
$$


Proof. It is easy to see that the equality

$$
(d-d \phi d)(x)=\sum_{\sigma^{(n-1)}} \sum_{\gamma \in \Gamma_{\sigma, \alpha}} m(\gamma)(x)
$$

follows from Lemma 5 when $x \in K_{\alpha}$.

The following lemma can sometimes be useful:

Lemma 6. The image of $\phi$ is given by

$$
\operatorname{Im}(\phi)=\bigoplus_{\alpha \in M^{+}} K_{\alpha}
$$

Proof. Let $\alpha \in M^{+}$with a vertex $\alpha \rightarrow \beta \in M$, and let $x \in K_{\alpha}$. We will prove that $x \in \operatorname{Im}(\phi)$ by induction on $\prec$. When $\alpha$ is a minimal element of $M^{+}$, it is easy to see that $\phi\left(d_{\beta, \alpha}(x)\right)=d_{\beta, \alpha}^{-1}\left(d_{\beta, \alpha}(x)\right)=x$. When $\alpha$ is non-minimal, we have that $x-\phi(d(x)) \in \bigoplus K_{\gamma}$, where the sum runs over all $\gamma \in M^{+}$with $\gamma \prec \alpha$, so, by induction, $x-\phi(d(x))=\phi(y)$ for some $y$, so $x=\phi(d(x))+\phi(y)$.

As a last result before dealing with the applications, we will see how one can, in certain situations when given a collection of Morse matchings on disjoint vertex sets, glue them together. Compare [Jon03, Lemma 2.2]. If $D=(V, E)$ is a digraph and $V^{\prime} \subseteq V$, we denote the induced subgraph on $V^{\prime}$ by $\left.D\right|_{V^{\prime}}$.

Lemma 7. Let $G_{\mathbf{K}}=(V, E)$ be the digraph of the based complex $\mathbf{K}$, and let $\sim$ be an equivalence relation on $V$ such that there is a well-founded partial order $\sqsubset$ on the equivalence classes satisfying $[\beta] \sqsubseteq[\alpha]$ whenever there is an edge $\alpha \rightarrow \beta$ in $G_{\mathbf{K}}$. Suppose $M_{[\alpha]}$ is a Morse matching on $\left.G_{\mathbf{K}}\right|_{[\alpha]}$ for all $[\alpha] \in V / \sim$. Then $\bigcup_{[\alpha]} M_{[\alpha]}$ is a Morse matching on $G_{\mathbf{K}}$.

Proof. For each equivalence class $[\alpha]$ there is a well-founded partial order $\prec_{[\alpha]}$. Define a partial order on the vertices $V$ by $\alpha \prec \beta$ if either $[\alpha] \sqsubset[\beta]$, or $[\alpha]=[\beta]$ and $\alpha \prec_{[\alpha]} \beta$. We will now show that $\prec$ is well founded. Suppose that $\left(\alpha_{i}\right)_{i \in \mathbb{N}}$ is a decreasing sequence (i.e. $\alpha_{i} \succeq \alpha_{i+1}$ for all $i$ ). Since $\sqsubset$ is well founded on $V / \sim$ there is an integer $N$ such that $\left[\alpha_{i}\right]=\left[\alpha_{N}\right]$ for all $i \geq N$. Since $\prec_{\left[\alpha_{i}\right]}$ is well founded, there is an $M>N$ such that $\alpha_{i}=\alpha_{M}$ for all $i \geq M$. From the well foundedness of $\prec$, the statement readily follows.

\section{Applications}

We will apply the theory to the computation of the homology of a certain family of finite-dimensional Lie algebras over a field of characteristic 2 . Then we will turn to the Anick resolution, which, we will see, can be easily constructed with the aid of a Morse matching in the bar resolution. Finally, using exactly the same matching we can construct a two-sided version of the Anick resolution, not previously in the literature.

3.1. The cohomology of a family of nilpotent Lie algebras. Let us first recall the construction of the Chevalley-Eilenberg complex, which, given a Lie algebra $\mathfrak{g}$ over a field $k$, gives a free resolution of the left $U(\mathfrak{g})$-module $k$. We will only consider finite-dimensional Lie algebras here, so we can assume that $\mathfrak{g}$ has a finite 
linearly-ordered basis $B$ as a vector space, with $|B|=N$. Consider the $k$-vector space $\bigwedge^{n} \mathfrak{g}$, which then has a basis consisting of all elements

$$
x_{1} \wedge x_{2} \wedge \cdots \wedge x_{n}, \quad x_{i} \in B, \quad x_{1}<x_{2}<\cdots<x_{n} .
$$

The Chevalley-Eilenberg resolution $\mathbf{V}$ is now given by

$$
0 \longrightarrow U(\mathfrak{g}) \otimes \bigwedge^{N} \mathfrak{g} \longrightarrow \cdots \longrightarrow U(\mathfrak{g}) \otimes \bigwedge^{2} \mathfrak{g} \longrightarrow U(\mathfrak{g}) \otimes \mathfrak{g} \longrightarrow U(\mathfrak{g}) \longrightarrow k \longrightarrow 0,
$$

where the differential is defined by

$$
\begin{aligned}
d\left(u \otimes x_{1} \wedge \cdots \wedge x_{n}\right)= & \sum_{i=1}^{n}(-1)^{i-1} u x_{i} \otimes x_{1} \wedge \cdots \wedge \widehat{x_{i}} \wedge \cdots \wedge x_{n} \\
& +\sum_{i<j}(-1)^{i+j} u \otimes\left[x_{i}, x_{j}\right] \wedge x_{1} \wedge \cdots \wedge \widehat{x_{i}} \wedge \cdots \wedge \widehat{x_{j}} \wedge \cdots \wedge x_{n} .
\end{aligned}
$$

The $p$ th homology (with trivial coefficients) of the Lie algebra $\mathfrak{g}$, denoted by $H_{p}(\mathfrak{g}, k)$, is equal to the $p$ th homology group of the complex $k \otimes_{U(\mathfrak{g})} \mathbf{V}$. An explicit description of the complex $k \otimes_{U(\mathfrak{g})} \mathbf{V}$ is

$$
0 \longrightarrow \bigwedge^{N} \mathfrak{g} \longrightarrow \bigwedge^{N-1} \mathfrak{g} \longrightarrow \cdots \longrightarrow \bigwedge^{2} \mathfrak{g} \longrightarrow \mathfrak{g} \longrightarrow k \longrightarrow 0
$$

with the differential

$$
\bar{d}\left(x_{1} \wedge \cdots \wedge x_{n}\right)=\sum_{i<j}(-1)^{i+j}\left[x_{i}, x_{j}\right] \wedge x_{1} \wedge \cdots \wedge \widehat{x_{i}} \wedge \cdots \wedge \widehat{x_{j}} \wedge \cdots \wedge x_{n} .
$$

We will now study the homologies of a certain family of Lie algebras, $\mathfrak{g}_{n}, n \geq 1$, which have been calculated by Armstrong, Cairns, and Jessup [ACJ97, when the ground field has characteristic 0 . In this section we will compute the homology of these Lie algebras when the ground field has characteristic 2. A basis for $\mathfrak{g}_{n}$ is $\left\{z, x_{1}, \ldots, x_{n}, y_{1}, \ldots, y_{n}\right\}$, and the non-zero brackets are $\left[z, x_{i}\right]=y_{i}$ for $1 \leq i \leq n$.

We will consider the Chevalley-Eilenberg complex with the basis elements $x_{I} \wedge$ $y_{J} \wedge u_{K}$ and $z \wedge x_{I} \wedge y_{J} \wedge u_{K}$, where $u_{i}=x_{i} \wedge y_{i}$ and $I, J, K$ are mutually disjoint subsets of $[n]$. Let us now define a subset $M$ of the edges in $G_{k \otimes \mathbf{V}}$ by

$$
M=\left\{z \wedge x_{I \cup\{j\}} \wedge y_{J} \wedge u_{K} \rightarrow x_{I} \wedge y_{J \cup\{j\}} \wedge u_{K} \mid I \cup J=\emptyset \text {, or } j<\min I \cup J\right\} .
$$

It is clear that $M$ is a partial matching, and furthermore, since for all vertices $\alpha$ and $\beta$ in $G_{k \otimes \mathbf{V}}$, such that $\alpha \rightarrow \beta \in M$ the corresponding component of the differential $d_{\beta, \alpha}$ clearly is an isomorphism, we only need to check the absence of directed cycles in $G_{k \otimes \mathbf{V}}^{M}$ to see that $M$ is a Morse matching. It should be clear that if we have a path

$$
x_{I} \wedge y_{J} \wedge u_{K} \rightarrow z \wedge x_{I^{\prime}} \wedge y_{J^{\prime}} \wedge u_{K^{\prime}} \rightarrow x_{I^{\prime \prime}} \wedge y_{J^{\prime \prime}} \wedge u_{K^{\prime \prime}}
$$

in $G_{k \otimes \mathbf{V}}^{M}$ where the two first vertices are matched, then $J$ and $J^{\prime \prime}$ are non-empty and $\min J^{\prime \prime}>\min J$. Similarly, if we have a path

$$
z \wedge x_{I} \wedge y_{J} \wedge u_{K} \rightarrow x_{I^{\prime}} \wedge y_{J^{\prime}} \wedge u_{K^{\prime}} \rightarrow z \wedge x_{I^{\prime \prime}} \wedge y_{J^{\prime \prime}} \wedge u_{K^{\prime \prime}}
$$

in $G_{k \otimes \mathbf{V}}^{M}$ where the two last vertices are matched, then $J$ and $J^{\prime \prime}$ are non-empty and $\min J^{\prime \prime}>\min J$. This shows that $M$ is a Morse matching.

The unmatched elements here are now of the following form:

$$
x_{I} \wedge y_{J} \wedge u_{K} \text {, where } I \cup J \neq \emptyset \text { and } \min (I \cup J) \in I \text {, or } I \cup J=\emptyset \text {, }
$$$$
z \wedge x_{I} \wedge y_{J} \wedge u_{K} \text {, where } I \cup J \neq \emptyset \text { and } \min (I \cup J) \in J \text {, or } I \cup J=\emptyset \text {. }
$$ 
The following theorem can be compared to the result of Armstrong, Cairns and Jessup ACJ97] that shows that when the characteristic of $k$ is 0 we have

$$
\operatorname{dim}_{k} H_{i}\left(\mathfrak{g}_{n}, k\right)=\left(\begin{array}{c}
n+1 \\
\left\lfloor\frac{i+1}{2}\right\rfloor
\end{array}\right)\left(\begin{array}{c}
n \\
\left\lfloor\frac{i}{2}\right\rfloor
\end{array}\right) .
$$

Theorem 3. When $k$ is a field of characteristic 2, the generating function of the Betti numbers of the Lie algebra $\mathfrak{g}_{n}$ is given by

$$
\sum_{i \geq 0} \operatorname{dim}_{k} H_{i}\left(\mathfrak{g}_{n}, k\right) z^{i}=\frac{1}{2}(1+z)\left((1+z)^{2 n}+\left(1+z^{2}\right)^{n}\right) .
$$

Proof. First we will show that the differential in the complex $\pi(k \otimes \mathbf{V})$ vanishes, which implies that $\operatorname{dim}_{k} H_{i}\left(\mathfrak{g}_{n}, k\right)$ is equal to the number of $M$-critical vertices in degree $i$, and then we will count the number of $M$-critical vertices.

So, let us assume that $z \wedge x_{I} \wedge y_{J \cup\{j\}} \wedge u_{K}$ is $M$-critical, with $j<\min (I \cup J)$. If $I=\emptyset$,

$$
d \pi\left(z \wedge x_{I} \wedge y_{J \cup\{j\}} \wedge u_{K}\right)=\pi d\left(z \wedge x_{I} \wedge y_{J \cup\{j\}} \wedge u_{K}\right)=0,
$$

so we consider the case when $I \neq \emptyset$. Since the characteristic of $k$ is 2 , we can safely ignore all signs in the following calculations, and we get:

$$
\begin{aligned}
& d \pi\left(z \wedge x_{I} \wedge y_{J \cup\{j\}} \wedge u_{K}\right)=d\left(z \wedge x_{I} \wedge y_{J \cup\{j\}} \wedge u_{K}\right)+d \phi d\left(z \wedge x_{I} \wedge y_{J \cup\{j\}} \wedge u_{K}\right) \\
& \quad=d\left(z \wedge x_{I} \wedge y_{J \cup\{j\}} \wedge u_{K}\right)+d \phi \sum_{i_{1} \in I} x_{I \backslash\left\{i_{1}\right\}} \wedge y_{J \cup\left\{i_{1}, j\right\}} \wedge u_{K} \\
& \quad=d\left(z \wedge x_{I} \wedge y_{J \cup\{j\}} \wedge u_{K}\right)+d \sum_{i_{1} \in I} z \wedge x_{I \backslash\left\{i_{1}\right\} \cup\{j\}} \wedge y_{J \cup\left\{i_{1}\right\}} \wedge u_{K} .
\end{aligned}
$$

Now

$$
d\left(z \wedge x_{I} \wedge y_{J \cup\{j\}} \wedge u_{K}\right)=\sum_{i_{1} \in I} x_{I \backslash\left\{i_{1}\right\}} \wedge y_{J \cup\left\{i_{1}, j\right\}} \wedge u_{K}
$$

and

$$
\begin{aligned}
d \sum_{i_{1} \in I} z \wedge x_{I \backslash\left\{i_{1}\right\} \cup\{j\}} \wedge y_{J \cup\left\{i_{1}\right\}} \wedge u_{K}= & \sum_{i_{1} \in I} x_{I \backslash\left\{i_{1}\right\}} \wedge y_{J \cup\left\{i_{1}, j\right\}} \wedge u_{K} \\
& +\sum_{\substack{i_{1}, i_{2} \in I \\
i_{1} \neq i_{2}}} x_{I \backslash\left\{i_{1}, i_{2}\right\} \cup\{j\}} \wedge y_{J \cup\left\{i_{1}, i_{2}\right\}} \wedge u_{K} \\
& =\sum_{i_{1} \in I} x_{I \backslash\left\{i_{1}\right\}} \wedge y_{J \cup\left\{i_{1}, j\right\}} \wedge u_{K} .
\end{aligned}
$$

Substituting the expressions (4) and (5) into the sum (3) finally gives

$$
d \pi\left(z \wedge x_{I} \wedge y_{J \cup\{j\}} \wedge u_{K}\right)=2 \sum_{i_{1} \in I} x_{I \backslash\left\{i_{1}\right\}} \wedge y_{J \cup\left\{i_{1}, j\right\}} \wedge u_{K}=0 .
$$

Since $d \pi\left(x_{I} \wedge y_{J} \wedge u_{K}\right)=\pi d\left(x_{I} \wedge y_{J} \wedge u_{K}\right)=0$ for all $I, J, K$, this implies that $d=0$ on $\pi(k \otimes \mathbf{V})$.

What now remains is to count the $M$-critical vertices. When $L \subseteq[n]$ and $K \subseteq[n] \backslash L$, the numbers of $M$-critical vertices of the form $x_{I} \wedge y_{J} \wedge u_{K}$ and of the form $z \wedge x_{I} \wedge y_{J} \wedge u_{K}$ with $I \cup J=L$ are both equal to $2^{|L|-1}$ if $L \neq \emptyset$, otherwise they are both equal to 1 . 
Thus they contribute $(1+z) 2^{|L|-1} z^{|L|+2|K|}$ when $L \neq \emptyset$, and $(1+z) z^{2|K|}$ otherwise. Finally, summing over $L$ and $K$ gives

$$
\begin{aligned}
\sum_{i \geq 0} \operatorname{dim}_{k} H_{i}\left(\mathfrak{g}_{n}, k\right) z^{i} & =\sum_{\emptyset \neq L \subseteq[n]} \sum_{K \subseteq[n] \backslash L}(1+z) 2^{|L|-1} z^{|L|+2|K|}+\sum_{K \subseteq[n]}(1+z) z^{2|K|} \\
& =\frac{1}{2}(1+z)\left(\sum_{\emptyset \neq L \subseteq[n]} \sum_{K \subseteq[n] \backslash L}(2 z)^{|L|}\left(z^{2}\right)^{|K|}+2 \sum_{K \subseteq[n]}\left(z^{2}\right)^{|K|}\right) \\
& =\frac{1}{2}(1+z)\left(\sum_{L \subseteq[n]} \sum_{K \subseteq[n] \backslash L}(2 z)^{|L|}\left(z^{2}\right)^{|K|}+\sum_{K \subseteq[n]}\left(z^{2}\right)^{|K|}\right) \\
& =\frac{1}{2}(1+z)\left(\left(1+2 z+z^{2}\right)^{n}+\left(1+z^{2}\right)^{n}\right) .
\end{aligned}
$$

3.2. The Anick resolution. Let $k$ be a field, let $X$ be a finite set of variables generating the free monoid $S$, and consider the non-commutative polynomial algebra $k\langle S\rangle$, viewed as the monoid algebra of $S$. The algebra $k\langle S\rangle$ is naturally graded with $k\langle S\rangle_{n}$ spanned by the words of length $n$ in $S$.

Let $I$ be a two-sided ideal in $k\langle S\rangle$ and let $A=k\langle S\rangle / I$. Let $\epsilon: A \rightarrow k$ be an augmentation, so $k$ is a left and right $A$-module. Anick [Ani86] has constructed an $A$-free resolution of $k$ as a right module. This resolution could be viewed as a deformation of Backelin's construction Bac78, which is an $A$-free resolution of $k$ in the case when $I$ is generated by monomials. We will see how an alternate description of Anick's resolution could easily be constructed using Morse theory.

First of all, we have to review the concept of an $n$-chain, which is vital in the construction of the resolution. Thus, let $W$ be a set of words in $S$ such that no word in $W$ is a subword of another word in $W$ (i.e. $W$ is an antichain with respect to the divisibility order in $S$ ). The two-sided ideal generated by $W$ in $k\langle S\rangle$ is denoted by $\langle W\rangle$.

Now, let us define a directed graph $G=(\mathcal{V}, \mathcal{E})$ with vertices $\mathcal{V} \subseteq S$, and edges $\mathcal{E} \subseteq \mathcal{V} \times \mathcal{V}$. We define $\mathcal{V}$ by

$$
\mathcal{V}=\{1\} \cup X \cup\{u \in S \mid u \text { is a proper right factor of some } v \in W\},
$$

and $\mathcal{E}$ by

(6) $\mathcal{E}=\{1 \rightarrow x \mid x \in X\}$

$$
\cup\{u \rightarrow v \mid u v \in\langle W\rangle, w \notin\langle W\rangle \text { for all proper left factors } w \text { of } u v\} .
$$

We now define $W^{(i)}$, the set of $i$-chains, for $i \geq-1$ by letting $W^{(i)}$ consist of the set of sequences $\left(v_{1}, \ldots, v_{i}, v_{i+1}\right)$ in $S^{i+1}$ such that there is a directed path $v_{0} v_{1} \cdots v_{i} v_{i+1}$ in $G$ with $v_{0}=1$. The use of the directed graph $G$ to describe the $i$-chains is due to Ufnarovski Ufn89]; see also [CPU99] for a discussion of the Anick resolution in terms of Gröbner bases.

Now, let $I$ be a two-sided ideal in $k\langle S\rangle$ and consider the quotient algebra $A=$ $k\langle S\rangle / I$. Let $\prec$ be a multiplicative well-order on $S$, and let $W$ be the minimal monomial generators of the initial ideal in $(I)$ (that is, the leading terms of a Gröbner basis for $I$ with respect to $\prec)$, and let $W^{(i)}$ be the corresponding chains. We will 
use the $k$-basis for $A$ that consists of the images of all monomials that are not members of in $(I)$.

Next, we will construct a Morse matching on the bar resolution of $k$, so we give a short review of the bar resolution: we define the complex $\mathcal{B}(k, A)$ with modules

$$
\mathcal{B}(k, A)_{n}=\bar{A}^{\otimes n} \otimes_{k} A=\bar{A} \otimes_{k} \cdots \otimes_{k} \bar{A} \otimes_{k} A, \quad n \text { copies of } \bar{A} .
$$

Here we use the notation $\bar{A}$ for the vector space cokernel of the map $k \rightarrow A$ given by $1 \mapsto 1$. The differential is defined in the standard manner by

$$
d\left(\left[a_{1}|\cdots| a_{n}\right]\right)=\sum_{i=1}^{n-1}(-1)^{i}\left[a_{1}|\cdots| a_{i} a_{i+1}|\cdots| a_{n}\right]+(-1)^{n}\left[a_{1}|\cdots| a_{n-1}\right] a_{n},
$$

where the notation $\left[a_{1}|\cdots| a_{n}\right]$ stands for $a_{1} \otimes \cdots \otimes a_{n} \otimes 1$.

We decompose $\mathcal{B}(k, A)_{n}$ as a direct sum of free $A$-modules in the following way:

$$
\mathcal{B}(k, A)_{n}=\bigoplus_{\substack{\left(w_{1}, \ldots, w_{n}\right) \in S_{+}^{n} \\ w_{i} \notin \operatorname{in}(I)}}\left[\bar{w}_{1}|\cdots| \bar{w}_{n}\right] \cdot A
$$

and we will write $\left(w_{1}, \ldots, w_{n}\right)$ instead of $\left[\bar{w}_{1}|\cdots| \bar{w}_{n}\right] \cdot A$ for the vertices in $G_{\mathcal{B}(k, A)}$.

For $\omega \in S$, let $V_{\omega, i}$ be the vertices $\left(w_{1}, \ldots, w_{n}\right)$ in $G_{\mathcal{B}(k, A)}$ such that $\omega=w_{1} \cdots w_{n}$ and $i$ is the largest integer $i \geq-1$ such that $\left(w_{1}, \ldots, w_{i+1}\right)$ is an $i$-chain. Let $V_{\omega}=\bigcup_{i} V_{\omega, i}$.

Define a partial matching $M_{\omega}$ on $\left(G_{\mathcal{B}(k, A)}\right)_{\omega}=\left.G_{\mathcal{B}(k, A)}\right|_{V_{\omega}}$ by letting $M_{\omega}$ consist of all edges

$$
\left(w_{1}, \ldots, w_{i+2}^{\prime}, w_{i+2}^{\prime \prime}, \ldots, w_{n}\right) \rightarrow\left(w_{1}, \ldots, w_{n}\right)
$$

when $\left(w_{1}, \ldots, w_{n}\right) \in V_{\omega, i}$, such that $w_{i+2}^{\prime} w_{i+2}^{\prime \prime}=w_{i+2}$ and $\left(w_{1}, \ldots, w_{i+1}, w_{i+2}^{\prime}\right)$ is an $(i+1)$-chain.

Lemma 8. The set of edges $M=\bigcup_{\omega} M_{\omega}$ is a Morse matching on $G_{\mathcal{B}(k, A)}$, with $M_{n}^{0}=W^{(n-1)}$ for all $n$.

Proof. We start by showing that $M_{\omega}$ is a Morse matching on the subgraph $\left(G_{\mathcal{B}(k, A)}\right)_{\omega}$ for all $\omega$.

First of all, $M_{\omega}$ is indeed a partial matching; it is easy to see that no vertex is the origin of more than one edge, and no vertex is the terminus of more than one edge either. The situation $\left(w_{1}, \ldots, w_{i+1}^{\prime}, w_{i+1}^{\prime \prime}, \ldots, w_{n}\right) \rightarrow\left(w_{1}, \ldots, w_{n}\right) \in M_{\omega}$, $\left(w_{1}, \ldots, w_{i+1}^{\prime}, w_{i+1}^{(3)}, w_{i+1}^{(4)}, \ldots, w_{n}\right) \rightarrow\left(w_{1}, \ldots, w_{i+1}^{\prime}, w_{i+1}^{\prime \prime}, \ldots, w_{n}\right) \in M_{\omega}$ cannot occur since this would imply that $w_{i+1}$ lies in in $(I)$.

Note that each $V_{\omega}$ is a finite set, so to prove that $M_{\omega}$ is a Morse matching, it is enough to prove that there are no directed cycles in $\left(G_{\mathcal{B}(k, A)}^{M}\right)_{\omega}$. Consider a vertex $v=\left(w_{1}, \ldots, w_{n}\right)$ in $\left(G_{\mathcal{B}(k, A)}\right)_{\omega}$, and look at the corresponding differential

$$
d\left(\left[w_{1}|\cdots| w_{n}\right]\right)=\sum_{i=1}^{n-1}(-1)^{i}\left[w_{1}|\cdots| w_{i} w_{i+1}|\cdots| w_{n}\right]+(-1)^{n}\left[w_{1}|\cdots| w_{n-1}\right] w_{n}
$$

The element $\left[w_{1}|\cdots| w_{n-1}\right] w_{n}$ is now in the component corresponding to the vertex $\left(w_{1}, \ldots, w_{n-1}\right)$ and $w_{1} \cdots w_{n-1} \prec w_{1} \cdots w_{n}$. The elements

$$
\left[w_{1}|\cdots| w_{i} w_{i+1}|\cdots| w_{n}\right]
$$


can all be written as linear combinations of elements in components corresponding to

$$
\left(w_{1}, \ldots, w_{i-1}, u, w_{i+2}, \ldots, w_{n}\right),
$$

where $w_{1} \cdots w_{i-1} u w_{i+2} \cdots w_{n} \preceq w_{1} \cdots w_{n}$, with equality or inequality depending on whether $w_{i} w_{i+1} \in \operatorname{in}(I)$ or not.

Now suppose we have a vertex $v=\left(w_{1}, \ldots, w_{n}\right)$ in $V_{\omega, i-1}$ and a directed path $v^{(n)} \rightarrow v_{1}^{(n+1)} \rightarrow v_{2}^{(n)}$ in $\left(G_{\mathcal{B}(k, A)}^{M}\right)_{\omega}$. Now

$$
v_{1}^{(n+1)}=\left(w_{1}, \ldots, w_{i+1}^{\prime}, w_{i+1}^{\prime \prime}, \ldots, w_{n}\right)
$$

with $\left(w_{1}, \ldots, w_{i}, w_{i+1}^{\prime}\right)$ an $i$-chain. Since $v_{2}^{(n)}$ is a vertex within the same component of the graph as $v^{(n)}$ and $v_{2}^{(n)} \neq v^{(n)}$, we must have that $v_{2}^{(n)}$ either is equal to

$$
\left(w_{1}, \ldots, w_{i+1}^{\prime}, w_{i+1}^{\prime \prime} w_{i+2}, \ldots, w_{n}\right)
$$

or to

$$
\left(w_{1}, \ldots, w_{i+1}^{\prime}, w_{i+1}^{\prime \prime}, \ldots, w_{j} w_{j+1}, \ldots, w_{n}\right)
$$

for some $j \geq i+2$. In both cases $v_{2}^{(n)}$ is in $V_{\omega, i}$, showing that there are no directed cycles in $\left(G_{\mathcal{B}(k, A)}^{M}\right)_{\omega}$.

So far we have thus shown that $M_{\omega}$ is a Morse matching on $\left(G_{\mathcal{B}(k, A)}\right)_{\omega}$, and since for every edge $u \rightarrow v$ from $u \in V_{\omega_{1}}$ to $v \in V_{\omega_{2}}$ we have $\omega_{2} \preceq \omega_{1}$, we can apply Lemma 7 and we have shown that $\bigcup_{\omega} M_{\omega}$ is a Morse matching.

The last thing we have to do is to determine $M^{0}$. Suppose $\left(w_{1}, \ldots, w_{n}\right) \in V_{\omega, i}$ is in $M^{0}$. We know that $\left(w_{1}, \ldots, w_{i+1}\right)$ is an $i$-chain.

Suppose $i<n-1$. Consider a vertex $\left(w_{1}, \ldots, w_{n}\right)$ in $V_{\omega, i}$ where the product $w_{i+1} w_{i+2} \in \operatorname{in}(I)$. Then there is a factorisation $w_{i+2}=w_{i+2}^{\prime} w_{i+2}^{\prime \prime}$ with $w_{i+2}^{\prime}$ minimal such that $w_{i+1} w_{i+2}^{\prime} \in \operatorname{in}(I)$. Since $\left(w_{1}, \ldots, w_{i+2}\right)$ is not an $(i+1)$-chain, $w_{i+2}^{\prime \prime} \neq 1$, which means that there is an edge

$$
\left(w_{1}, \ldots, w_{i+2}^{\prime}, w_{i+2}^{\prime \prime}, \ldots, w_{n}\right) \rightarrow\left(w_{1}, \ldots, w_{n}\right)
$$

in $M$. Thus $\left(w_{1}, \ldots, w_{n}\right) \notin M^{0}$.

Next, consider $\left(w_{1}, \ldots, w_{n}\right)$ in $V_{\omega, i}$ where $w_{i+1} w_{i+2} \notin \operatorname{in}(I)$. Now, there is an edge $\left(w_{1}, \ldots, w_{n}\right) \rightarrow\left(w_{1}, \ldots, w_{i+1} w_{i+2}, \ldots, w_{n}\right)$ in $M$, which means $\left(w_{0}, \ldots, w_{n}\right) \notin$ $M^{0}$. Since it is obvious that all vertices $\left(w_{1}, \ldots, w_{n+1}\right)$ in $V_{\omega, n}$ are in $M^{0}$, we conclude that $M_{n+1}^{0}$ consists precisely of the $n$-chains.

Thus we can describe the Anick resolution $\mathbf{F}$ as the following: let $F_{n}=W^{(n-1)} \otimes_{k}$ $A$ and define the differential $d_{n}$ as follows: for an $(n-1)$-chain $\omega=\left(w_{1}, \ldots, w_{n}\right)$, let $i: W^{(n-1)} \otimes A \rightarrow \mathcal{B}(k, A)_{n}$ be defined by $i\left(w_{1}, \ldots, w_{n}\right)=\left[w_{1}|\cdots| w_{n}\right]$ and $p: \mathcal{B}(k, A)_{n} \rightarrow W^{(n-1)}$ on the basis elements by $p\left(\left[w_{1}|\cdots| w_{n}\right]\right)=\left(w_{1}, \ldots, w_{n}\right) \otimes 1$ if $\left(w_{1}, \ldots, w_{n}\right)$ is an $n$-chain and $p\left(\left[w_{1}|\cdots| w_{n}\right]\right)=0$ otherwise. Now,

$$
d_{n}=p\left(d-d \phi_{M} d\right) i \text {. }
$$

As a direct consequence of the preceding lemma and Theorem 2 we now get the following:

Theorem 4 ([Ani86, Theorem 1.4]). The complex $\mathbf{F}$ defined above is a free $A$ resolution of $k$. 
The Anick resolution is implemented in the Gröbner basis program Bergman Bac. In Anick's paper, the differential $d_{n}$ is defined in terms of $d_{n-1}$ when $n>2$, which is not the case for the definition using the Morse matching. This could possibly be a computational advantage if one wants to calculate a specific homology group using the Anick resolution.

3.3. A two-sided Anick resolution. The approach used to derive the Anick resolution can equally well be applied to derive a resolution for an algebra over its enveloping algebra. Keeping the notation from the previous section, we shall see how to construct a free $A^{\mathrm{e}}$-resolution of $A$ as a left module.

Here we consider the two-sided bar resolution $\mathcal{B}(A, A)$ which is an $A^{\mathrm{e}}$-free resolution of $A$ where

$$
\mathcal{B}(A, A)_{n}=A \otimes_{k} \bar{A}^{\otimes n} \otimes_{k} A \simeq A^{\mathrm{e}} \otimes_{k} \bar{A}^{\otimes n} .
$$

The differential is defined similarly as before:

$$
\begin{aligned}
d\left(\left[a_{1}|\cdots| a_{n}\right]\right)= & a_{1}\left[a_{2}|\cdots| a_{n}\right] \\
& +\sum_{i=1}^{n-1}(-1)^{i}\left[a_{1}|\cdots| a_{i} a_{i+1}|\cdots| a_{n}\right] \\
& +(-1)^{n}\left[a_{1}|\cdots| a_{n-1}\right] a_{n} .
\end{aligned}
$$

As before, we decompose the modules in the resolution as follows:

$$
\mathcal{B}(A, A)=\bigoplus_{\substack{\left(w_{1}, \ldots, w_{n}\right) \in S_{+}^{n} \\ w_{i} \notin \operatorname{in}(I)}} A^{\mathrm{e}} \cdot\left[\bar{w}_{1}|\cdots| \bar{w}_{n}\right],
$$

and we consider the same matching $M=\bigcup_{\omega} M_{\omega}$ as before, and get

Lemma 9. The set of edges $M=\bigcup_{\omega} M_{\omega}$ is a Morse matching on $G_{\mathcal{B}(A, A)}$, with $M_{n}^{0}=W^{(n-1)}$ for all $n$.

Proof. The digraph $G_{\mathcal{B}(A, A)}$ has the same vertex set as $G_{\mathcal{B}(k, A)}$, and the edge set of $G_{\mathcal{B}(A, A)}$ is the union of the edge set of $G_{\mathcal{B}(k, A)}$ with a set of edges of the form

$$
\left(w_{1}, \ldots, w_{n}\right) \rightarrow\left(w_{2}, \ldots, w_{n}\right) .
$$

Since $w_{2} \cdots w_{n} \prec w_{1} \cdots w_{n}$ the proof of Lemma 8 can be reused.

Similarly as before (using the notation from Theorem 4), we define a complex G where $G_{n}=A^{\mathrm{e}} \otimes_{k} W^{(n-1)}$, where the differential is given using the maps $i$ and $p$ from Theorem 4

$$
d_{n}=p\left(d-d \phi_{M} d\right) i
$$

Theorem 5. The complex $\mathbf{G}$ defined above is a free $A^{\mathrm{e}}$-resolution of $A$.

This result generalises Bardzell's resolution for monomial algebras Bar97; however, in this case Bardzell has found a much simpler form for the differential. 


\section{REFERENCES}

[ACJ97] Grant F. Armstrong, Grant Cairns, and Barry Jessup, Explicit Betti numbers for a family of nilpotent Lie algebras, Proc. Amer. Math. Soc. 125 (1997), no. 2, 381-385. MR97d:17013

[Ani86] David J. Anick, On the homology of associative algebras, Trans. Amer. Math. Soc. 296 (1986), no. 2, 641-659. MR87i:16046

[Bac] Jörgen Backelin, The Gröbner basis calculator Bergman, Available at http://www.math.su.se/bergman/.

[Bac78] Jörgen Backelin, La série de Poincaré-Betti d'une algèbre graduée de type fini à une relation est rationnelle, C. R. Acad. Sci. Paris Sér. A-B 287 (1978), no. 13, A843-A846. MR:81f:16002

[Bar97] Michael J. Bardzell, The alternating syzygy behavior of monomial algebras, J. Algebra 188 (1997), no. 1, 69-89. MR98a:16009

[BBL:99] Eric Babson, Anders Björner, Svante Linusson, John Shareshian, and Volkmar Welker, Complexes of not i-connected graphs, Topology 38 (1999), no. 2, 271-299. MR2000a:57001

[BL91] Donald W. Barnes and Larry A. Lambe, A fixed point approach to homological perturbation theory, Proc. Amer. Math. Soc. 112 (1991), no. 3, 881-892. MR91j:55019

[BW02] E. Batzies and V. Welker, Discrete Morse theory for cellular resolutions, J. Reine Angew. Math. 543 (2002), 147-168. MR2003b:13017

[Cha00] Manoj K. Chari, On discrete Morse functions and combinatorial decompositions, Discrete Math. 217 (2000), no. 1-3, 101-113, Formal power series and algebraic combinatorics (Vienna, 1997). MR2001g:52016

[CPU99] Svetlana Cojocaru, Alexander Podoplelov, and Victor Ufnarovski, Non-commutative Gröbner bases and Anick's resolution, Computational methods for representations of groups and algebras (Essen, 1997), Birkhäuser, Basel, 1999, pp. 139-159. MR2000i:16090

[For98] Robin Forman, Morse theory for cell complexes, Adv. Math. 134 (1998), no. 1, 90-145. MR99b:57050

[Jon03] Jakob Jonsson, On the topology of simplicial complexes related to 3-connected and Hamiltonian graphs, J. Combin. Theory Ser. A 104 (2003), no. 1, 169-199. MR2018427 (2004h:05130)

[JW05] Michael Jöllenbeck and Volkmar Welker, Resolution of the residue class field via algebraic discrete morse theory, arXiv:math.AC/0501179, 2005.

[Sha01] John Shareshian, Discrete Morse theory for complexes of 2-connected graphs, Topology 40 (2001), no. 4, 681-701. MR2002h:57017

[Ufn89] V. A. Ufnarovskiı, On the use of graphs for calculating the basis, growth and Hilbert series of associative algebras, Mat. Sb. 180 (1989), no. 11, 1548-1560, 1584. MR91d:16053

Department of Mathematics, National University of Ireland, Galway, Ireland

E-mail address: emil.skoldberg@nuigalway.ie

$U R L$ : www. nuigalway.ie/maths/es/ 